ONLINE MUTATION REPORT

\title{
Hypofibrinogenaemia caused by a novel FGG missense mutation (W253C) in the $\gamma$ chain globular domain impairing fibrinogen secretion
}

\author{
D Vu, P de Moerloose, A Batorova, J Lazur, L Palumbo, M Neerman-Arbez
}

J Med Genet 2005;42:e57 (http://www.jmedgenet.com/cgi/content/full/42/9/e57). doi: 10.1136/jmg.2005.033530

\begin{abstract}
Background: Inherited disorders of fibrinogen are rare and affect either the quantity (hypofibrinogenaemia and afibrinogenaemia) or the quality of the circulating fibrinogen (dysfibrinogenaemia). Extensive allelic heterogeneity has been found for all three disorders: in congenital afibrinogenaemia $>30$ mutations, the majority in FGA, have been identified in homozygosity or in compound heterozygosity. Several mutations have also been identified in patients with hypofibrinogenaemia; many of these are heterozygous carriers of afibrinogenaemia null mutations.

Objective: To report the case of a patient from Slovakia diagnosed with hypofibrinogenaemia characterised by fibrinogen concentrations of around $0.7 \mathrm{~g} / \mathrm{l}$.

Results: The patient was found to be heterozygous for a novel missense mutation W253C (W227C in the mature protein) in the $\mathrm{C}$-terminal globular domain of the fibrinogen $\gamma$ chain. Co-expression of the W253C FGG mutant cDNA (fibrinogen Bratislava) in combination with wild-type FGA and FGB CDNAs showed that fibrinogen molecules containing the mutant $\gamma$ chain can assemble intracellularly but are not secreted into the media, confirming the causative nature of the identified mutation.

Conclusions: Current analysis of fibrinogen Bratislava indicates that the domains important for the processes of hexamer assembly and hexamer secretion should not be considered as strictly restricted to one or other fibrinogen chain.
\end{abstract}

$\mathrm{F}$ ibrin is the major protein of the blood clot and is necessary for the mechanical binding of platelets and other plasma proteins, forming a network of fibrin polymers to prevent blood flow from an injured vessel. Fibrin is produced following proteolytic cleavage of fibrinogen by thrombin which allows polymerisation to occur. ${ }^{1}$ Fibrinogen is a $340 \mathrm{kDa}$ glycoprotein predominantly synthesised by hepatocytes; it is composed of two sets of three homologous polypeptide chains known as $\mathrm{A} \alpha-$, $\mathrm{B} \beta-$-, and $\gamma$ chains, which assemble to form a hexameric structure $(\mathrm{A} \alpha \mathrm{B} \beta \gamma)_{2}$. Each polypeptide is encoded by a distinct gene, $F G A, F G B$, and $F G G$, respectively, clustered in a region of $50 \mathrm{~kb}$ on chromosome $4 \mathrm{q}^{3} 1 .^{2}$

Fibrinogen is normally found in plasma at concentrations of between 1.5 and $3.5 \mathrm{~g} / \mathrm{l}$. Inherited disorders of fibrinogen are rare and affect either the quantity (hypofibrinogenaemia and afibrinogenaemia) or the quality of the circulating fibrinogen (dysfibrinogenaemia). More than 400 cases of dysfibrinogenaemia have been reported to date, the first dysfibrinogenaemia mutation being identified as early as $1968 .^{3}$ The majority of dysfibrinogenaemias are caused by missense mutations in one of the three fibrinogen genes. Missense mutations at residue $\mathrm{R} 35$ ( $\mathrm{R} 35 \mathrm{H}$ and $\mathrm{R} 35 \mathrm{C}$, or $\mathrm{R} 16 \mathrm{H}$ and $\mathrm{Rl6C}$ in the mature protein), which is part of the thrombin cleavage site in the fibrinogen $\alpha$ chain, are the most common. In contrast, the molecular basis of afibrinogenaemia/hypofibrinogenaemia has only recently become elucidated.

Congenital afibrinogenaemia is characterised by complete deficiency of fibrinogen. In 1999, we identified the first causative mutations for congenital afibrinogenaemia: the genetic defect in a non-consanguineous Swiss family was an apparently recurrent deletion of approximately $11 \mathrm{~kb}$ of DNA which eliminates the majority of the FGA gene and so leads to a complete absence of functional fibrinogen. ${ }^{4}$ Since our identification of the disease locus, numerous causative mutations have been characterised in $F G A$ (the great majority of cases ${ }^{5}$ ) but also in $F G G$ and $F G B$, allowing a precise molecular diagnosis for the patients as well as prenatal diagnosis for the families concerned.

Of particular interest are missense mutations leading to complete fibrinogen deficiency: four such missense mutations have been described in FGB: L383R, G430D, ${ }^{7} \mathrm{G} 444 \mathrm{~S},{ }^{8}$ and $\mathrm{W} 467 \mathrm{G},{ }^{9}$ numbered from the initiator ATG. These missense mutations all lie in the $\mathrm{C}$ terminal portion of the fibrinogen $\beta$ chain, which is highly conserved among vertebrates. In addition, two nonsense mutations in the same region were recently characterised. The first, W470X, was identified in heterozygosity in an asymptomatic patient. ${ }^{10}$ The second nonsense mutation, W467X, three codons upstream, was identified in homozygosity in two Palestinian sisters with afibrinogenaemia and results in a fibrinogen $\beta$ chain which lacks only the last 25 amino acid residues. ${ }^{11}$ Expression studies in transfected cells performed for the four missense and the W467X $\beta$ chain C-terminal mutations both in our laboratory and by others showed that an intact $\beta$ C-terminal domain is necessary for hexamer secretion into the circulation, although assembly inside the cell appears unimpaired. Interestingly, this property of the fibrinogen $\beta$ chain seems to differ from the C-terminus of the fibrinogen $\gamma$ chain, which has been shown to be necessary for intracellular hexamer assembly in experiments investigating serial deletions of the $\gamma \mathrm{C}$-terminus, ${ }^{12}$ but also in studies on hypofibrinogenaemia mutants such as fibrinogen Matsumoto IV. ${ }^{13} 14$

Congenital hypofibrinogenaemia is characterised by functional and antigenic fibrinogen levels lower than $1.5 \mathrm{~g} / \mathrm{l}$. Although in the past hypofibrinogenaemia was considered a separate disorder from afibrinogenaemia, with both dominant and recessive modes of inheritance proposed, recent studies (reviewed by Maghzal et $a l^{6}$ ) have shown that in

Abbreviations: ATG, annotated translation start codon; $\mathrm{CHO}$, Chinese hamster ovary 
many cases patients are asymptomatic and are in fact heterozygous for null mutations which in homozygosity or compound heterozygosity would cause afibrinogenaemia.

In this study, a patient with hypofibrinogenaemia was found to be heterozygous for a novel missense mutation in $F G G:$ TGG $\rightarrow$ TGT, W253C (W227 in the mature protein) in exon 7. The localisation of this missense mutation close to the previously reported mutations Matsumoto $\mathrm{IV}^{13}$ and Hakata ${ }^{15}$ prompted us to carry out co-transfection experiments in COS7 cells in order to assay its consequence on fibrinogen assembly or secretion and to confirm its causative nature.

\section{METHODS}

\section{Clinical description}

The patient, a woman now aged 38, with no family history of bleeding, was diagnosed with hypofibrinogenaemia at the age of 22 years after a bleeding complication of delivery. Her father and mother died at the age of 70 and 63 years, from myocardial infarction and a brain tumour, respectively. The patient suffered from frequent epistaxis during childhood, mild bleeding after tooth extractions, and prolonged wound bleeding and healing, none however requiring therapeutic intervention. Menarche and subsequent menstrual bleedings were normal. Hypofibrinogenaemia was diagnosed on the basis of low fibrinogen levels $(0.7 \mathrm{~g} / \mathrm{l})$ after the first spontaneous delivery was complicated by serious bleeding and the development of a large pararectal haematoma. The patient received three units of red blood cells and a surgical revision was made after an infusion of $3.0 \mathrm{~g}$ of fibrinogen concentrate (Fibrinogen, Immuno, Vienna, Austria). At the age of 28 years she had a missed abortion in the first trimester. Mild bleeding occurred again at the age of 31 years after a second spontaneous delivery managed by replacement of 3.0 grams of fibrinogen, which increased the circulating fibrinogen to $1.6 \mathrm{~g} / \mathrm{l}$. At the age of 38 years the patient underwent laparoscopic surgery after preoperative administration of $3.0 \mathrm{~g}$ fibrinogen, without any bleeding complications.

\section{Coagulation studies}

Prothrombin time (PT), activated partial thromboplastin time (aPTT), thrombin time (TT), and functional factors II, V, VII, VIII, IX, X, and von Willebrand antigen were measured using reagents, calibrators, and factor deficient plasmas from Dade Behring (Marburg, Germany). Fibrin/fibrinogen degradation products levels were determined with Thrombo-Welcotest (Biotech, Murex, Dartford, UK) and D-dimer with DD-Plus (Dade Behring, Berlin, Germany). Clottable fibrinogen was measured with the Clauss method and fibrinogen antigen with Turbiquant fibrinogen using a Turbitimer ${ }^{\circledR}$ (both from Dade Behring). Bleeding time was measured according to Duke, and the euglobulin lysis time (ELT) by standard methods. After obtaining informed consent from the patient, blood was collected in EDTA for DNA analysis.

\section{Mutation screening}

All exons and intron-exon junctions of the $F G A, F G G$, and $F G B$ genes were amplified by polymerase chain reaction (PCR) and sequenced as previously described. ${ }^{516}$

\section{Expression and analysis of the mutations in COS-7 cells}

Construction of wild type and mutant expression vectors, transient co-transfections of COS-7 cells, cell lysate and conditioned media harvesting, and western blot analysis were carried out essentially as previously described, ${ }^{17}$ with some minor modifications. The forward oligonucleotides used for site-directed mutagenesis were: 5'-GCACAACAG AATTTTGTCTGGGAAATGAGAAG-3' for the Trp253Cys substitution (highlighted in bold); 5'-CCACTATGAAGATATAG CCATTCAACAGACTCAC-3' for Ile413stop, 5' -GAAGAAAACC ACTATGTAGATAATCCCATTCAAC-3' for Lys4llstop, and 5'-CGGTGGTATTCCATGTAGAAAACCACTATG-3' for Lys406stop with the inserted stop codon in bold. The whole cDNA sequence of all mutants was confirmed by sequencing. Twenty four hours after transfection, cells were washed with phosphate buffered saline (PBS) and incubated for an

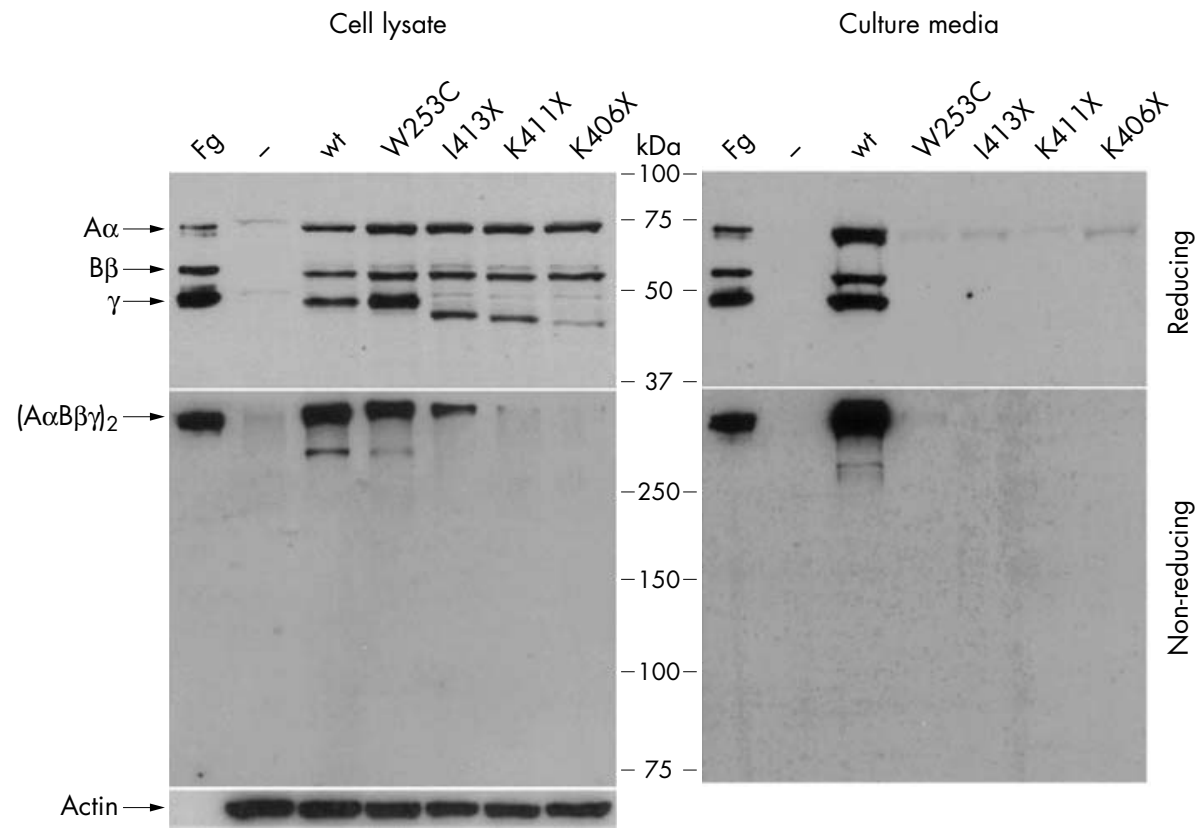

Figure 1 Western blot analysis of cell extracts and conditioned media of COS-7 cells transfected with fibrinogen cDNAs. Samples of cell lysates and culture medium were subjected to 10\% SDS-PAGE under reducing conditions or 7\% SDS-PAGE under non-reducing conditions. The blots were incubated with a polyclonal antihuman fibrinogen antibody and cross reacting bands revealed by chemiluminescence. Fg, purified fibrinogen control; , $C O S$ cells transfected with the empty vector. The positions of the hexameric complex and the normal $A \alpha, B \beta$, and $\gamma$ chains are indicated. Loading control was carried out with antiactin antibodies on reduced cell lysates. 


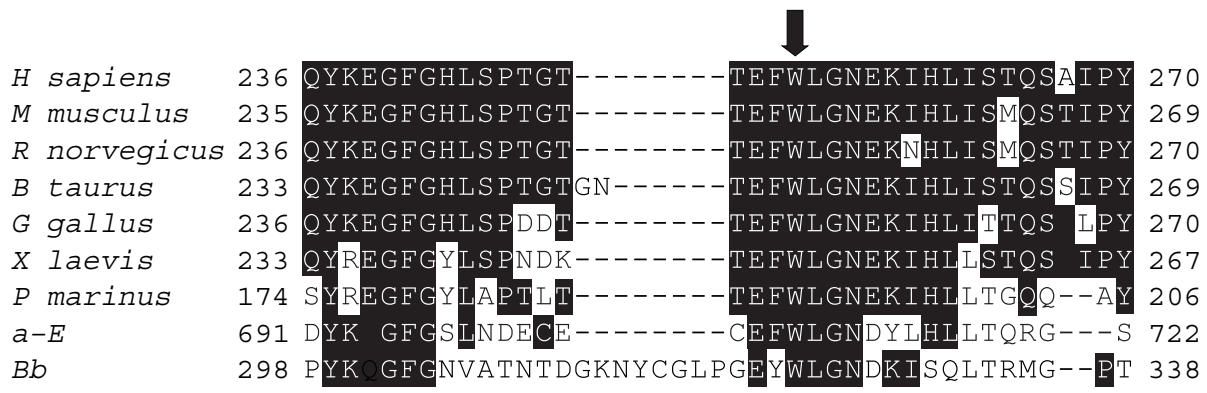

Figure 2 Multiple alignment of fibrinogen $\gamma$ chain and of human fibrinogen $\alpha E$ and $B \beta$ chains in the region surrounding the human W253 residue. Human (P02679-2), mouse (Q8VCM7; from TrEMBL), rat (P02680-2), cow (P12799), chicken (NP_990320; from NCBI), Xenopus (P17634), and lamprey (P041 15) fibrinogen $\gamma$ sequences, as well as human fibrinogen $\alpha \mathrm{E}$ (P02671) and B $\beta$ sequences (P02675) were obtained from the Swiss-Prot database, if not otherwise indicated, and aligned using the ClustalW program (http://www.ebi.ac.uk/clustalw/). Residue numbering is from the first ATG codon. Identical amino acids are shaded black. The human W253 residue is indicated by the arrow.

additional 24 hours in media without serum. Conditioned medium with added proteases inhibitors (Complete, Roche) was concentrated using Amicon Ultra-4 $5 \mathrm{kDa}$ (Millipore) and cells were scraped in Ripa buffer before addition of reducing or non-reducing $4 \times$ Laemmli buffer.
Independent experiments were repeated three times. Western blot analysis was undertaken using rabbit antihuman fibrinogen antibodies (DakoCytomation) at a 1:2500 or 1:1500 dilution, or mouse antiactin antibodies (1:2500; Chemicon).

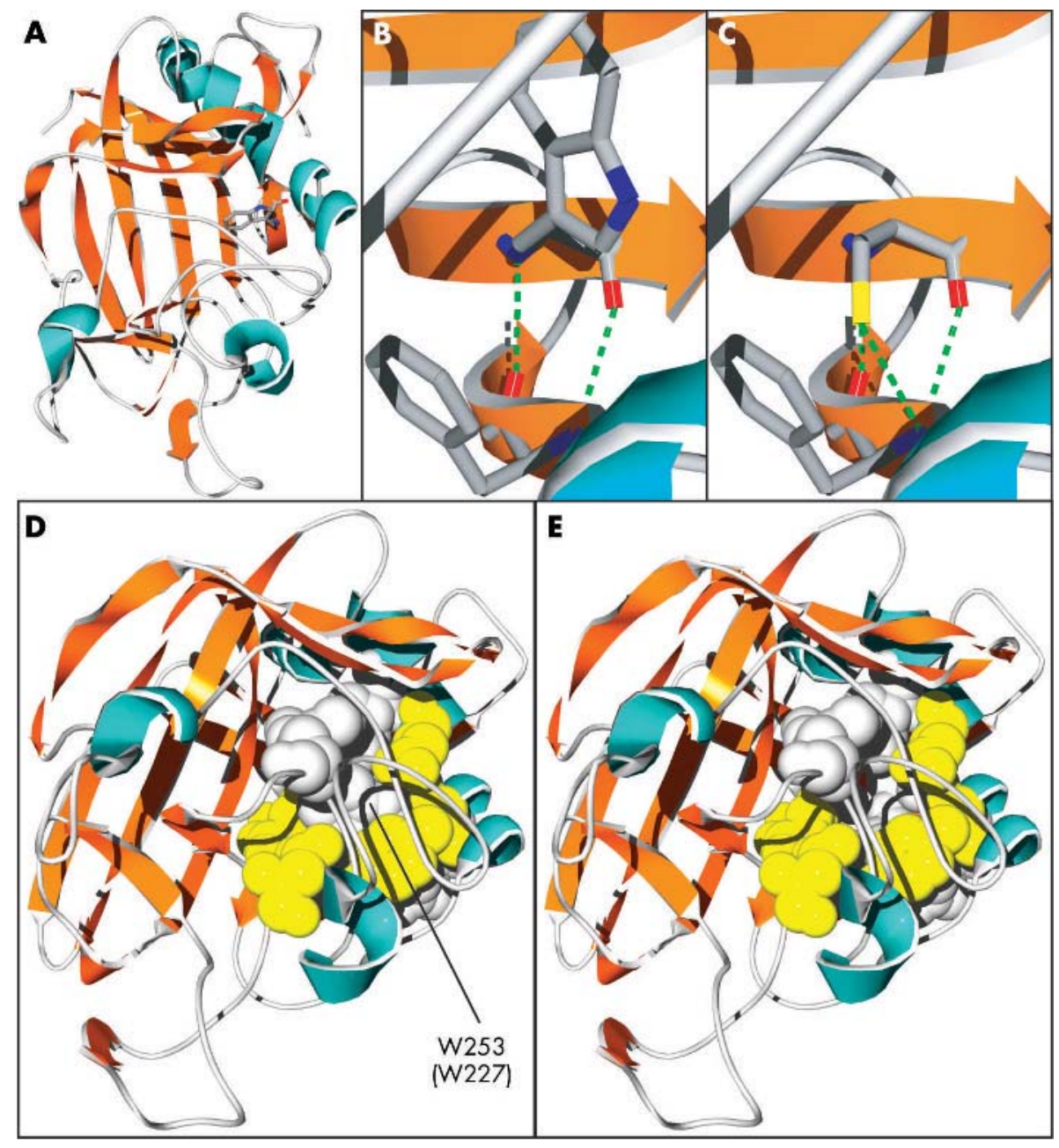

Figure 3 Crystallographic structure analysis of the $\gamma D$ domain. (A) View of the $\gamma$ C-terminal fragment with the position of W253 (W227 in the mature protein) in the central antiparallel $\beta$ sheet, with helix highlighted in green, and $\beta$ strands in orange. (B) W253 (W227) forms two H-bonds (green) with F241 (F215 in the mature protein), while (C) the W253C mutation may create a third H-bond. (D) W253 (W227) is buried and surrounded by polar (yellow) and non-polar (grey) amino acids, within a 4 Å radius. (E) Replacement with a cysteine residue creates a void, possibly leading to local refolding. Figures were prepared using Swiss-PdbViewer and POV-Ray from the PDB file 1FIB. 


\section{RESULTS AND DISCUSSION}

The patient presented as a classical case of hypofibrinogenaemia with a proportional decrease in fibrinogen coagulant and antigen levels ( $0.7 \mathrm{~g} / \mathrm{l}$ and $0.8 \mathrm{~g} / \mathrm{l}$, respectively). She also had a prolonged thrombin time $(31 \mathrm{~s}$, normal range $16-21 \mathrm{~s}$ ). The aPTT was also slightly prolonged (ratio 1.25, normal range 0.8 to 1.2 ), while PT, other coagulation factor levels, and vWF Ag were within the normal range.

Despite moderate fibrinogen deficiency the patient experienced several bleeding episodes as well as a spontaneous abortion, which was not explained by examination of the placenta or by genetic analysis of the fetus. The bleeding episodes were either spontaneous (epistaxis in childhood) or provoked (tooth extractions, cut wounds, postpartum). The initial course of the first spontaneous delivery was normal; the development of a large pararectal haematoma was provoked by the episiotomy probably potentiated by hypofibrinogenaemia, with rapid lysis of the weak fibrin clot. Haemotherapy with three units of RBC, plasma, and $3.0 \mathrm{~g}$ fibrinogen prevented the bleeding complications of surgical revision of the haematoma. However, mild bleeding occurred again following the second spontaneous delivery without episiotomy, despite preventive administration of $3.0 \mathrm{~g}$ fibrinogen which resulted in a circulating fibrinogen level of $1.6 \mathrm{~g} / \mathrm{l}$. In contrast, preoperative infusion of the same dose of fibrinogen was sufficient to prevent the bleeding complication after laparoscopic surgery carried out six years later. This supports the suggestion that injury or surgical interventions on parenchymous organs with physiologically high fibrinolytic potential may require more intensive fibrinogen replacement therapy in patients with hypofibrinogenaemia and a history of a bleeding tendency.

Sequence analysis of genomic DNA revealed no mutation in either FGA or FGB; in contrast, a novel missense mutation$\mathrm{TGG} \rightarrow \mathrm{TGT}$, W253C (fibrinogen Bratislava)—was identified in $F G G$ exon 7. The localisation of this missense mutation close to the previously reported mutations Matsumoto $\mathrm{IV}^{13}$ and Hakata ${ }^{15}$ prompted us to undertake co-transfection experiments in COS-7 cells in order to assay its effects on fibrinogen assembly and secretion and confirm its causative nature.

The FGG W253C mutant CDNA, obtained by site directed mutagenesis of the wild-type $F G G$ cDNA as described in Methods, was transiently co-transfected in COS-7 cells with wild-type $F G A$ and $F G B$ cDNAs. Three negative controls for intracellular hexamer assembly were also produced and analysed in the same way. These were FGG I413X, K411X, and K406X, corresponding to a deletion of 25, 27, and 32 residues, respectively, from the $\gamma$ chain $\mathrm{C}$-terminus. ${ }^{12}$ Forty eight hours after transfection, individual fibrinogen chains (under reducing conditions) or assembled hexamers (under non-reducing conditions) in cell extracts and conditioned media were detected by western blot analysis with a polyclonal antifibrinogen antibody.

Co-transfection with the three normal cDNAs showed expression of all three polypeptides, normal assembly inside the cell, and adequate secretion of the hexamer (fig 1). When co-transfections were undertaken with wild-type $F G A$ and $F G B$ cDNAs in combination with the mutant FGG W253C CDNA, the three polypeptides were expressed and were able to assemble intracellularly to form apparently normal hexamers. In contrast, co-expression of wild-type $F G A$ and $F G B$ with mutant $F G G$ W253C cDNA abolished the secretion into the media of the mutant $\gamma$ chain, as well as the mutant containing hexamer.

Surprisingly, under these conditions-when co-transfections were carried out with wild type $F G A$ and $F G B$ cDNAs and with the mutant I413X FGG, which we had designed as a negative control for hexamer assembly-a band corresponding to the hexamer was detectable in cell lysates, although this was always fainter than the wild type band. This result appears to contradict data from a recent study ${ }^{12}$ showing that no hexamer is detected when this truncated $\gamma$ chain is cotransfected in CHO cells. In order to assess whether such differences could be attributable to a greater permissiveness of COS cells to incorporate truncated $\gamma$ chains into hexamers, we analysed two more severely truncated variants: K411X and K406X. In both cases, the hexamer was absent or barely detectable. These data confirm the validity of our in vitro transfected COS-7 cell system for distinguishing between defects in fibrinogen assembly and secretion, even though it is not identical to the CHO model.

Residue W253 (W227 in the mature protein) is absolutely conserved in seven species from lamprey to human, as well as in the human fibrinogen $\alpha \mathrm{E}$ and $\beta$ chains (fig 2). In the $\gamma$ chain, it is surrounded by many hydrophobic residues and buried in a central $\beta$ strand (fig 3A). The crystallographic structure of the $\gamma$ chain suggests that the backbone of W253 (W227) forms two H-bonds with the backbone of F24l (F215 in the mature protein, fig $3 \mathrm{~B}$ ). Substitution of $\mathrm{W}$ to $\mathrm{C}$ is predicted to add another $\mathrm{H}$-bond between both residues (fig 3C). Furthermore the replacement of $\mathrm{W}$ by the smaller $\mathrm{C}$ may create a void leading to local refolding in order to close the gap (fig 3D, 3E), possibly destabilising the normal folding of the $\gamma \mathrm{C}$ domain and causing the non-secretion of fibrinogen hexamers. Interestingly, in the fibrinogen molecule there appears to be no potential partner for disulphide bridge formation with the new cysteine residue within a radius of $10 \AA$. Nevertheless, it is conceivable that the novel free - $\mathrm{SH}$ group interacts with reactive sulphydryl groups of other proteins present in the cell-for example, albumin, particularly in the endoplasmic reticulum.

The study of this novel mutation provides new insight into the process of fibrinogen assembly and secretion. Indeed, mutations specifically inhibiting fibrinogen secretion (and not assembly) have previously been identified in the Cterminal portion of the $\beta$ chain, ${ }^{7-9}{ }^{11}$ suggesting a preferential role of this domain as a target for quality control of hexamer secretion. In contrast, in a study of serial deletions of the $\gamma$ chain, truncation of the last $25 \mathrm{C}$-terminal residues or more was shown to prevent the assembly of the hexamer in $\mathrm{CHO}$ cells. ${ }^{12}$ Similarly the $\gamma$ C179R Matsumoto IV variant fails to form $A \alpha / \gamma$ and $B \beta / \gamma$ complexes, which are the normal assembly intermediates of fibrinogen. ${ }^{14}$

Interpretation of these observations might lead to the simple conclusion that the $\beta \mathrm{C}$-terminal domain is critical for fibrinogen secretion, while the $\gamma \mathrm{C}$-terminal domain is essential for fibrinogen assembly. Our current analysis of fibrinogen Bratislava, together with other $\gamma$ mutants such as fibrinogen Brescia ${ }^{18}$ G310R and Aguadilla ${ }^{19}$ R401W-which also abolish secretion and also cause an intriguing hepatic endoplasmic reticulum storage disease-indicates that the domains important for the processes of hexamer assembly and hexamer secretion should not be considered as being restricted to one or other fibrinogen chain.

\section{ACKNOWLEDGEMENTS}

We thank Holger Scheib for helpful comments on the crystallographic analysis. This study was supported by Swiss National Science Foundation (SNF) grant No 31-64987.01, by the Téléthon Action Suisse Foundation, the Ernst and Lucie Schmidheiny Foundation and a Bayer Hemophilia Special Project award. MNA is the recipient of an SNF professorship (No 631-66023).

\section{Authors' affiliations}

D Vu, L Palumbo, M Neerman-Arbez, Department of Genetic Medicine and Development, University Medical School, Geneva, Switzerland P de Moerloose, Division of Angiology and Haemostasis, University Hospital, Geneva 
A Batorova, National Haemophilia Centre, Department of Haematology, University Hospital, Bratislava, Slovakia

J Lazur, Department of Haematology, University Hospital, Kosice, Slovakia

Competing interests: MN-A received a Bayer Haemophilia Project Award.

Correspondence to: Professor Marguerite Neerman-Arbez, Centre Médical Universitaire, 1 rue Michel-Servet, $\mathrm{CH}-1211$ Geneva, Switzerland; Marguerite.Arbez@medecine.unige.ch

Revised version received 9 May 2005

Accepted for publication 10 May 2005

\section{REFERENCES}

1 Mosesson MW, Siebenlist KR, Meh DA. The structure and biological features of fibrinogen and fibrin. Ann NY Acad Sci 2001;936:11-30.

2 Kant JA, Fornace AJ, Saxe D, Simon MI, McBride OW, Crabtree GR. Evolution and organization of the fibrinogen locus on chromosome 4: gene duplication accompanied by transposition and inversion. Proc Natl Acad Sci USA 1985;82:2344-8.

3 Blomback M, Blomback B, Mammen EF, Prasad AS. Fibrinogen Detroit - a molecular defect in the $\mathrm{N}$-terminal disulphide knot of human fibrinogen? Nature 1968;218:134-7.

4 Neerman-Arbez M, Honsberger A, Antonarakis SE, Morris MA. Deletion of the fibrinogen alpha-chain gene (FGA) causes congenital afibrinogenemia. J Clin Invest 1999;103:215-18.

5 Neerman-Arbez M, de Moerloose P, Bridel C, Honsberger A, Schonborner A, Rossier C, Peerlinck K, Claeyssens S, Di Michele D, d'Oiron R, Dreyfus M, Laubriat-Bianchin M, Dieval J, Antonarakis SE, Morris MA. Mutations in the fibrinogen aalpha gene account for the majority of cases of congenital afibrinogenemia. Blood 2000;96:149-52.

6 Maghzal GJ, Brennan SO, Homer VM, George PM. The molecular mechanisms of congenital hypofibrinogenaemia. Cell Mol Life Sci 2004;61:1427-38.

7 Duga S, Asselta R, Santagostino E, Zeinali S, Simonic T, Malcovati M, Mannucci PM, Tenchini ML. Missense mutations in the human beta fibrinogen gene cause congenital afibrinogenemia by impairing fibrinogen secretion. Blood 2000;95:1336-41.

8 Vu D, Bolton-Maggs PH, Parr JR, Morris MA, de Moerloose P, NeermanArbez $M$. Congenital afibrinogenemia: identification and expression of a missense mutation in FGB impairing fibrinogen secretion. Blood 2003;102:4413-15.

9 Spena S, Asselta R, Duga S, Malcovati M, Peyvandi F, Mannucci PM, Tenchini ML. Congenital afibrinogenemia: intracellular retention of fibrinogen due to a novel W437G mutation in the fibrinogen Bbeta-chain gene. Biochim Biophys Acta 2003;1639:87-94

10 Homer VM, Brennan SO, Ockelford P, George PM. Novel fibrinogen truncation with deletion of Bbeta chain residues 440-461 causes hypofibrinogenaemia. Thromb Haemost 2002:88:427-31.

11 Neerman-Arbez M, Vu D, Abu-Libdeh B, Bouchardy I, Morris MA. Prenatal diagnosis for congenital afibrinogenemia caused by a novel nonsense mutation in the FGB gene in a Palestinian family. Blood 2003;101:3492-4.

12 Okumura N, Terasawa F, Tanaka H, Hirota M, Ota H, Kitano K, Kiyosawa K, Lord ST. Analysis of fibrinogen gamma-chain truncations shows the Cterminus, particularly gammalle387, is essential for assembly and secretion of this multichain protein. Blood 2002;99:3654-60.

13 Terasawa F, Okumura N, Kitano K, Hayashida N, Shimosaka M, Okazaki M, Lord ST. Hypofibrinogenemia associated with a heterozygous missense mutation gamma153Cys to arg (Matsumoto IV): in vitro expression demonstrates defective secretion of the variant fibrinogen. Blood 1999;94:4122-31.

14 Terasawa F, Fujita K, Okumura N. Residue gamma153Cys is essential for the formation of the complexes Aalphagamma and Bbetagamma, assembly intermediates for the AalphaBbetagamma complex and intact fibrinogen. Clin Chim Acta 2005;353:157-64

15 lida $H$, Ishii $E$, Nakahara $M$, et al. A case of congenital afibrinogenemia: fibrinogen Hakata, a novel nonsense mutation of the fibrinogen gamma-chain gene. Thromb Haemost 2000;84:49-53.

16 Neerman-Arbez M de Moerloose P, Honsberger A, Parlier G, Arnuti B, Biron C, Borg JY, Eber S, Meili E, Peter-Salonen K, Ripoll L, Vervel C, d'Oiron R, Staeger P, Antonarakis SE, Morris MA. Molecular analysis of the fibrinogen gene cluster in 16 patients with congenital afibrinogenemia: novel truncating mutations in the FGA and FGG genes. Hum Genet 2001;108:237-40.

17 Neerman-Arbez M, Germanos-Haddad M, Tzanidakis K, Vu D, Deutsch S, David A, Morris MA, de Moerloose P. Expression and analysis of a split premature termination codon in FGG responsible for congenital afibrinogenemia: escape from RNA surveillance mechanisms in transfected cells. Blood 2004;104:3618-23.

18 Brennan SO, Wyatt J, Medicina D, Callea F, George PM. Fibrinogen brescia: hepatic endoplasmic reticulum storage and hypofibrinogenemia because of a gamma284 Gly $\rightarrow$ Arg mutation. Am J Pathol 2000;157:189-96.

19 Brennan SO, Maghzal G, Shneider BL, Gordon R, Magid MS, George PM. Novel fibrinogen gamma375 Arg $\rightarrow \operatorname{Trp}$ mutation (fibrinogen aguadilla) causes hepatic endoplasmic reticulum storage and hypofibrinogenemia. Hepatology 2002;36:652-8. 